\title{
Forward Time Centered Space Scheme for the Solution of Transport Equation
}

\author{
Jawdat Alebraheem * \\ Mathematics Department, College of Science Al Zufli, Majmaah University, Saudi Arabia \\ * Corresponding author email: j.alebraheem@mu.edu.sa
}

Received: 15 March 2017 / Revised: 28 March 2017 / Accepted: 07 April 2017 / Published: 09 April 2017

\begin{abstract}
Transport phenomenon is central for understanding many procedures in numerous sciences, transport phenomena can be described by the partial differential equation. Forward Time Centered Space scheme give ample numerical solutions of the transport equation. In this paper, we apply Forward Time Centered Space scheme to solve a non-trivial transport problem using different step sizes of time $(\mathrm{t})$ and space $(\mathrm{x})$. We use MATLAB software to get the numerical results. The numerical simulation presents that the FTCS scheme is more stable and closer to the exact solution when we decrease step sizes of $t, x$ and $\alpha$ more and more.
\end{abstract}

Keywords: Transport equation; Forward Time Centered Space scheme; numerical simulations.

\section{Introduction}

The transport phenomenon is a central for understanding many procedures in numerous sciences as engineering, agriculture, meteorology, physiology, biology, analytical chemistry, materials science, pharmacy, and other areas. Transport phenomenon is a well-developed and very useful branch of physics that used in many areas of applied science [1]. Transport equation describes many transport phenomena such as heat transfer, mass transfer, fluid dynamics, spread of neutrons, sound propagation, and other applications of physics [2]. Differential equations have been applied to describe many natural phenomena in many areas [3] - [8]. Transport equation that has different forms is a general partial differential equation. One dimensional transport equation that we use in this paper is: $\frac{\partial T}{\partial t}+u \frac{\partial T}{\partial x}-\alpha \frac{\partial^{2} T}{\partial x^{2}}=0$

where $\mathrm{T}$ is a passive scalar (e.g. temperature concentration) which is being transported with a known velocity $\mathrm{u}(\mathrm{x}, \mathrm{t})$. If $\mathrm{T}$ is the temperature, then $\alpha$ is called the thermal diffusivity coefficient. The development in computer hardware and numerical methods algorithms has led to use numerical methods in solving many applied problems. Therefore, the recent years have witnessed shifting from analytical techniques to computer-oriented numerical methods. The primary appeal of numerical methods is that they provide solutions for many problems, which are not amenable through analytical solution. The development in modern digital computers paved the way for the development of efficient finite difference 
methods, which are employed for solving particularly partial differential equations [9].

Finite difference methods present sufficient numerical results in a simpler and more efficient way. Finite difference methods are used for solving partial differential equation which gives the required solutions while analytical methods are often fail [10]. In this paper, we apply FTCS (Forward Time Centered Space) scheme that is an example of explicit finite difference scheme to solve a non-trivial transport problem which has sharp continuous initial condition.

\section{Forward Time Centred Space Scheme}

For using FTCS scheme to solve the equation (1) we take the forward time and central space at point $\left(x_{j}, t_{n}\right)$, and apply them on the equation

(1) [11]. So we obtain:

$\frac{T_{j}^{n+1}-T_{j}^{n}}{\Delta t}+\frac{u\left(T_{j+1}^{n}-T_{j-1}^{n}\right)}{2 \Delta x}-\frac{\alpha\left(T_{j-1}^{n}-2 T_{j}^{n}+T_{j+1}^{n}\right)}{\Delta x^{2}}=0$

The equation (2) can be written as an algorithm: $T_{j}^{n+1}=(s+0.5 C) T_{j-1}^{n}+(1-2 s) T_{j}^{n}+(s-0.5 C) T_{j+1}^{n}$

Where $\mathrm{s}=\frac{\alpha \Delta t}{\Delta x^{2}}$ and $\mathrm{C}=\frac{u \Delta t}{\Delta x}$.

We can write equation (3) for proposal of programming as follows:

$T_{j}^{n}=(s+0.5 C) T_{j-1}^{n-1}+(1-2 s) T_{j}^{n-1}+(s-0.5 C) T_{j+1}^{n-1}$

According to the Von Neuman stability analysis; the stability condition for FTCS scheme is as follows [12]:

$$
0 \leq C^{2} \leq 2 s \leq 1 \text {. }
$$

The local truncation error for FTCS scheme is [11]:

$$
C u\left(\frac{\Delta x}{2}\right) \frac{\partial^{2} T}{\partial x^{2}}-\left[C \alpha \Delta x-u\left(\frac{\Delta x^{2}}{6}\right)\left(1+2 C^{2}\right)\right] \frac{\partial^{3} T}{\partial x^{3}}
$$

\section{Analytical Solution}

The separation of variables technique is used to get the exact solution for equation (1), it was obtained by Fletcher [11], as follows:

$$
T(x, t)=0.5-\frac{2}{\pi} \sum_{k=1}^{N} \sin \left[(2 k-1) \frac{\pi(x-u t)}{L}\right] \frac{\exp \left[-\alpha(2 k-1)^{2} \pi^{2} t / L^{2}\right]}{2 k-1}
$$

We indicate that the initial and boundary conditions are:

$\mathrm{T}(\mathrm{x}, 0)=1.0$ if $-2 \leq x<0$ (initial condition) (8) $\mathrm{T}(\mathrm{x}, 0)=0.0$ if $0 \leq x \leq 2 \quad$ (initial condition) (9) $\mathrm{T}(-2, \mathrm{t})=1.0$ if $t>0$ (boundary condition) (10) $\mathrm{T}(2, \mathrm{t})=0.0$ if $t>0$ (boundary condition)

\section{Numerical Simulation Results}

Numerical simulations are carried out using MATLAB program to obtain FTCS scheme results for transport equation at specific values of space $(\mathrm{x})$ that are $\{-2.0,-1.5,-1.0,-0.5,0.0,0.5$, $1.0,1.5,2.0\}$ when the value of time at $t=1.0$, for different values of $\Delta x, \Delta t$ and $\alpha$.

We use a FTCS scheme formula (3), the exact solution formula (7) and the initial and boundary conditions to compare between FTCS scheme and the exact solution results.

\subsection{FTCS Scheme Results (when $\Delta x=0.5$,}

$$
\Delta t=0.1 \text { and } \alpha=1.0 \text { ) }
$$

To run the MATLAB program, we assume in the first case the following values $(\Delta x=0.5$, $\Delta t=0.1, u=1.0, \alpha=1.0$ and $L=4$ ), to obtain the results of FTCS scheme for transport equation and exact solution for transport equation. We pick $\Delta x=0.5$ to generate the all values of $x$ that are $\{-2.0,-1.5,-1.0,-0.5,0.0,0.5$, $1.0,1.5,2.0\}$, we select $\Delta t=0.1$ to get the value of time when $t=1.0, L=4$ represents the length of axis $\mathrm{x}(-2 \leq x \leq 2)$ which represents the boundary for the problem, and we select $u=1.0$ and $\alpha=1.0$ to satisfy the stability condition in addition to the values of $\Delta x$ and $\Delta t$ We consider these values as a first case to be:-

$s=0.4$ where $s=\frac{\alpha \Delta t}{\Delta x^{2}}$

and $C=0.2$ where $\mathrm{C}=\frac{u \Delta t}{\Delta x}$.

We select these values that they satisfy the condition (5). We use specific values of space $(\mathrm{x})$ that are $\{-2.0,-1.5,-1.0,-0.5,0.0,0.5,1.0,1.5,2.0\}$ 
Jawdat Alebraheem., Int. Ann. Sci.; Vol. 2, Issue 1, pp: 1-5, 2017

when the value of time at $t=1.0$ to get the following table:

Table 1: Results of FTCS scheme with Exact solution for transport equation when $\Delta x=0.5$, $\Delta t=0.1$ and $\alpha=1.0$

\begin{tabular}{|c|c|c|c|}
\hline Value of $x$ & $\begin{array}{c}\text { FTCS } \\
\text { Scheme }\end{array}$ & $\begin{array}{c}\text { Exact } \\
\text { Solution }\end{array}$ & $\begin{array}{c}\text { Absolute } \\
\text { Error }\end{array}$ \\
\hline-2.0 & 1.0000 & 1.0000 & 0.0000 \\
\hline-1.5 & 0.9625 & 0.8171 & 0.1454 \\
\hline-1.0 & 0.9045 & 0.8427 & 0.0618 \\
\hline-0.5 & 0.8213 & 0.8171 & 0.0042 \\
\hline 0.0 & 0.7104 & 0.7435 & 0.0331 \\
\hline 0.5 & 0.5759 & 0.6322 & 0.0563 \\
\hline 1.0 & 0.4205 & 0.5000 & 0.0795 \\
\hline 1.5 & 0.2410 & 0.3678 & 0.1268 \\
\hline 2.0 & 0.0000 & 0.0000 & 0.0000 \\
\hline
\end{tabular}

We notice from the Table 1 that the sum of absolute error is 0.5071 and the average of absolute error is 0.05634 .

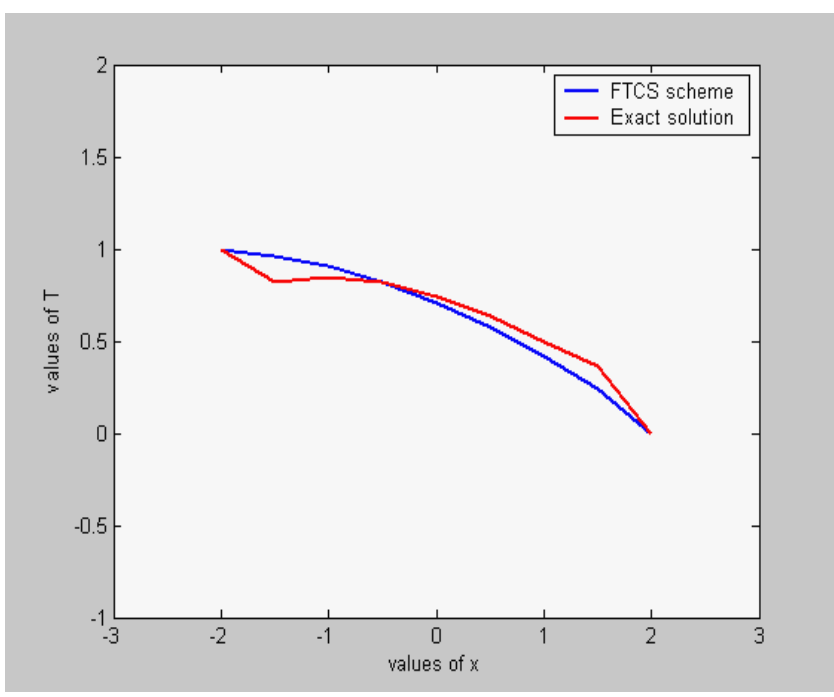

Figure 1: FTCS scheme and Exact solution of transport equation together when $\Delta x=0.5$, $\Delta t=0.1$ and $\alpha=1.0$

Figure 1 describes the results of FTCS scheme with exact solution together for transport equation at specific values of space $(\mathrm{x})$ which are $\{-2.0,-1.5,-1.0,-0.5,0.0,0.5,1.0,1.5,2.0\}$ and the value of time at $t=1.0$ when $\Delta x=0.5$ $\Delta t=0.1$ and $\alpha=1.0$
4.2 FTCS Scheme Results (when $\Delta x=0.25$,

$$
\Delta t=0.05 \text { and } \alpha=0.5 \text { ) }
$$

To run the MATLAB program in the second case, we suppose the following values ( $\Delta x=0.25, \Delta t=0.05, u=1.0, \alpha=0.5$ and $L=4$ ), to obtain the results in this case of FTCS scheme for transport equation and exact solution for transport equation. We pick $\Delta x=0.25$ to generate the all values of $x$ that are $\{-2.0,-1.5$, $1.0,-0.5,0.0,0.5,1.0,1.5,2.0\}$, we select $\Delta t=0.05$ to get the value of time when $t=1.0$ , $L=4$ represents the length of axis $\mathrm{x}$ ( $-2 \leq x \leq 2$ ) which represents the boundary for the problem, and we select $u=1.0$ and $\alpha=0.5$ to satisfy the stability condition (5) in addition to the values of $\Delta x$ and $\Delta t$.

We consider these values as a second case to be-

$s=0.4$ Where $\mathrm{s}=\frac{\alpha \Delta t}{\Delta x^{2}}$

and $C=0.2$ where $\mathrm{C}=\frac{u \Delta t}{\Delta x}$.

We select these values so that they satisfy the condition (5). We use specific values of space (x) which are $\{-2.0,-1.5,-1.0,-0.5,0.0,0.5,1.0,1.5$, $2.0\}$ when $t=1.0$ to get the following table according to second case:

Table 2: Results of FTCS scheme with exact solution of transport equation when $\Delta x=0.25, \Delta t=0.05$ and $\alpha=0.5$

\begin{tabular}{|c|c|c|c|}
\hline $\begin{array}{c}\text { Value of } \\
\mathrm{x}\end{array}$ & $\begin{array}{c}\text { FTCS } \\
\text { Scheme }\end{array}$ & $\begin{array}{c}\text { Exact } \\
\text { Solution }\end{array}$ & $\begin{array}{c}\text { Absolute } \\
\text { Error }\end{array}$ \\
\hline-2.0 & 1.0000 & 1.0000 & 0.0000 \\
\hline-1.5 & 0.9926 & 0.9270 & 0.0656 \\
\hline-1.0 & 0.9712 & 0.9545 & 0.0167 \\
\hline-0.5 & 0.9186 & 0.9270 & 0.0084 \\
\hline 0.0 & 0.8149 & 0.8400 & 0.0251 \\
\hline 0.5 & 0.6533 & 0.6912 & 0.0379 \\
\hline 1.0 & 0.4537 & 0.5000 & 0.0463 \\
\hline 1.5 & 0.2491 & 0.3088 & 0.0597 \\
\hline 2.0 & 0.0000 & 0.0000 & 0.0000 \\
\hline
\end{tabular}

We notice from the Table 2 that the sum of absolute error is 0.2597 and the average of 
absolute error is 0.02886 . We notice that the results of FTCS scheme for transport equation in the second case are more accurate than the first case because $\Delta x=0.25, \Delta t=0.05$ and $\alpha=0.5$ is used in the second case while $\Delta x=0.5, \Delta t=0.1$ and $\alpha=1.0$ is used in the first case. This means the results of FTCS scheme for transport equation in the second case is more improve than the results of FTCS scheme for transport equation in the first case.

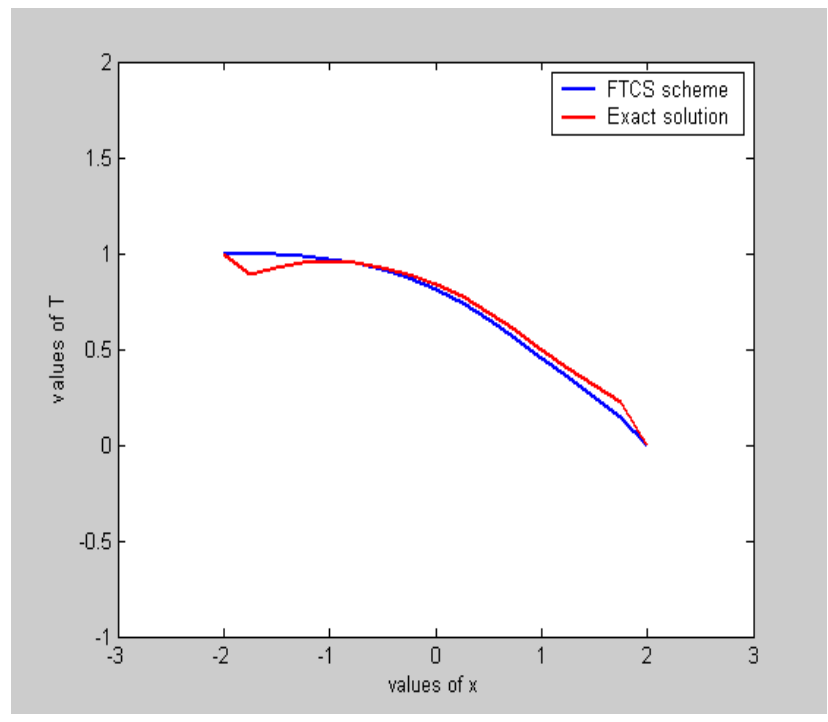

Figure 2: FTCS scheme and Exact solution together of transport equation when $\Delta x=0.25$,

$$
\Delta t=0.05 \text { and } \alpha=0.5
$$

The Figure 2 describes the results of FTCS scheme with exact solution together for transport equation at specific values of space $(\mathrm{x})$ which are $\{-2.0,-1.5,-1.0,-0.5,0.0,0.5,1.0,1.5,2.0\}$ and the value of time at $t=1.0$ when $\Delta x=0.25$ $\Delta t=0.05$ and $\alpha=0.5$.

\subsection{FTCS Scheme Results (when $\Delta \mathrm{x}=0.05$,}

$$
\Delta t=0.01 \text { and } \alpha=0.0625 \text { ) }
$$

To run the MATLAB program in third case, we suppose the following values $(\Delta \mathrm{x}=0.05$, $\Delta t=0.01, u=1.0, \alpha=0.0625$ and $L=4)$, to obtain the results in this case of FTCS scheme for transport equation and exact solution of transport equation. We pick $\Delta x=0.25$ to generate the all values of $\mathrm{x}$ that are $\{-2.0,-1.5$, $1.0,-0.5,0.0,0.5,1.0,1.5,2.0\}$, we select $\Delta t=0.05$ to get the value of time when $t=1.0$, we choose $L=4$ represents the length of axis $\mathrm{x}(-2 \leq x \leq 2)$ which represents the boundary for the problem, and we select $u=1.0$ and $\alpha=0.5$ to satisfy the stability condition (5) in addition to the values of $\Delta x$ and $\Delta t$.

We consider these values as a third case to be:-

$s=0.25$ Where $s=\frac{\alpha \Delta t}{\Delta x^{2}}$

and $C=0.2$ where $C=\frac{u \Delta t}{\Delta x}$.

We select these values to satisfy the condition (5). We also use specific values of space $(\mathrm{x})$ that are $\{-2.0,-1.5,-1.0,-0.5,0.0,0.5,1.0,1.5,2.0\}$ when $t=1.0$ to get the following third case table:

Table 3: Results of FTCS scheme with Exact solution of transport equation when $\Delta \mathrm{x}=0.05$,

$$
\Delta t=0.01 \text { and } \alpha=0.0625
$$

\begin{tabular}{|c|c|c|c|}
\hline $\begin{array}{c}\text { Value of } \\
\mathrm{x}\end{array}$ & $\begin{array}{c}\text { FTCS } \\
\text { Scheme }\end{array}$ & $\begin{array}{c}\text { Exact } \\
\text { Solution }\end{array}$ & $\begin{array}{c}\text { Absolute } \\
\text { Error }\end{array}$ \\
\hline-2.0 & 1.0000 & 1.0000 & 0.0000 \\
\hline-1.5 & 1.0000 & 1.0000 & 0.0000 \\
\hline-1.0 & 1.0000 & 1.0000 & 0.0000 \\
\hline-0.5 & 1.0000 & 1.0000 & 0.0000 \\
\hline 0.0 & 0.9966 & 0.9977 & 0.0011 \\
\hline 0.5 & 0.8946 & 0.9214 & 0.0268 \\
\hline 1.0 & 0.4142 & 0.5000 & 0.0858 \\
\hline 1.5 & 0.0440 & 0.0786 & 0.0346 \\
\hline 2.0 & 0.0000 & 0.0000 & 0.0000 \\
\hline
\end{tabular}

We notice from the Table 3 that the sum of absolute error is 0.1483 and the average of absolute error is 0.016478 . We notice that the results of FTCS scheme for transport equation in the third case are more accurate than those of the first case and the second case because $\Delta x=0.05$, $\Delta t=0.01$ and $\alpha=0.0625$ are used in the third case, while $\Delta x=0.25, \Delta t=0.05$ and $\alpha=0.5$ are used in the second case and in the first case $\Delta x=0.5, \Delta t=0.1$ and $\alpha=1.0$ are used. This means the results of FTCS scheme for transport equation in the third case are the most improve the results of FTCS scheme for transport equation in the first case, and in the second case. It is noticed that whenever $\Delta x, \Delta t$ and $\alpha$ 
Jawdat Alebraheem., Int. Ann. Sci.; Vol. 2, Issue 1, pp: 1-5, 2017

decrease, the sum and the average of absolute error in FTCS scheme decrease, this means that the results become more accurate and close to the exact solution whenever $\Delta x, \Delta t$ and $\alpha$ are small.

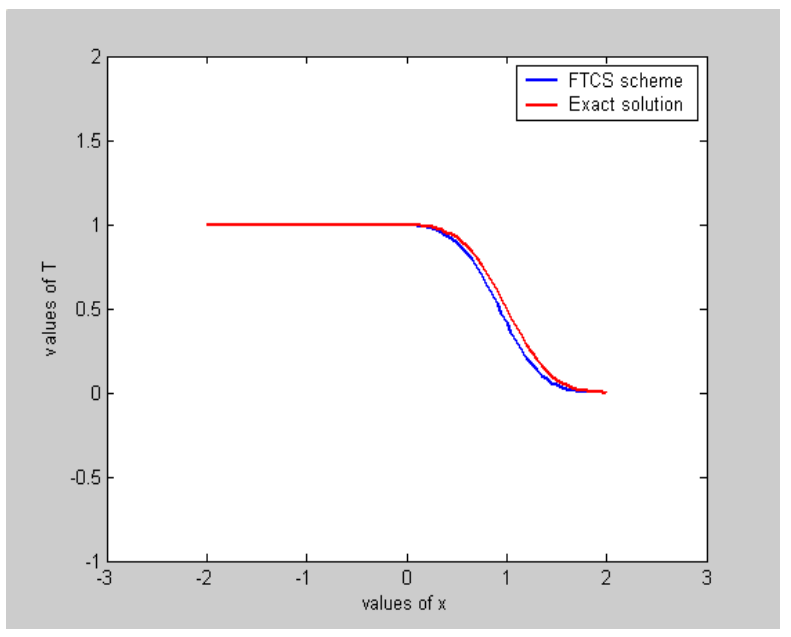

Figure 3: FTCS scheme and Exact solution together of transport equation when $\Delta \mathrm{x}=0.05$,

$$
\Delta t=0.01 \text { and } \alpha=0.0625
$$

The Figure 3 describes the results of FTCS scheme with exact solution for transport equation at specific values of space $(\mathrm{x})$ that are $\{-2.0,-1.5$, $-1.0,-0.5,0.0,0.5,1.0,1.5,2.0\}$ and the value of time at $t=1.0$ when $\Delta \mathrm{x}=0.05, \Delta t=0.01$ and $\alpha=0.0625$.

\section{Conclusions}

Transport equation has been considered for describing transport phenomena in many areas, that is partial differential equation. We have applied Forward Time Centred Space scheme to solve a non-trivial transport problem using different step sizes of time $(\mathrm{t})$ and space $(\mathrm{x})$. MATLAB software has been used to get the results. The numerical simulations presents three different values of $\Delta x, \Delta t$ and $\alpha$ with fixing other parameter values at specific values of space (x) that are $\{-2.0,-1.5,-1.0,-0.5,0.0,0.5,1.0,1.5$, $2.0\}$ when the value of time at $t=1.0$. the results show FTCS scheme is more stable and closer to the exact solution through the absolute error when we decrease step sizes of $\mathrm{t}, \mathrm{x}$ and $\alpha$ more and more.

\section{How to Cite this Article:}

J. Alebraheem, "Forward Time Centered Space Scheme for the Solution of Transport Equation", International Annals of Science, vol. 2, no. 1, pp. 1-5, 2017. doi: 10.21467/ias.2.1.1-5

\section{References}

[1] B. R. Bird, E. W. Stewart, and N. E. Lightfoot. "Transport Phenomena". Second Edition. John Wiley and Sons, New York, 2002.

[2] V.I Agoshkov."Boundary Value Problems for Transport Equations". Birkhauser Boston. Institute of Numerical Mathematics, RAS, Moscow, 1998.

[3] J. Alebraheem, Y. Abu-Hassan, "Persistence of Predators in a Two Predators- One Prey Model with NonPeriodic Solution", Applied Mathematical Sciences, Vol. 6, No. 19, 943-956, 2012.

[4] J. Alebraheem and Y. Abu-Hassan. "Efficient Biomass Conversion and its Effect on the Existence of Predators in a Predator-Prey System", Research Journal of Applied Sciences, Vol. 8, 286-295, 2013.

[5] J. Alebraheem and Y. Abu-Hassan. "The Effects of Capture Efficiency on the Coexistence of a Predator in a Two Predators-One Prey Model", Journal of Applied Sciences, Vol. 11, 3717-3724, 2011.

[6] J. Alebraheem and Y. Abu-Hassan. "Dynamics of a two predator-one prey system". Computational and Applied Mathematics, 1-14, 2013.

[7] J. Alebraheem and Y. Abu-Hassan, "Simulation of complex dynamical behavior in prey predator model," 2012 International Conference on Statistics in Science, Business and Engineering (ICSSBE), Langkawi, pp. 1-4, 2012.

[8] M. Lysaker, A. Lundervold and Xue-Cheng Tai, "Noise removal using fourth-order partial differential equation with applications to medical magnetic resonance images in space and time," in IEEE Transactions on Image Processing, vol. 12, no. 12, pp. 1579-1590, Dec. 2003.

[9] Rao. S. K. "Numerical Methods for Scientists and Engineers". Third Edition. Prentice-Hall of India, New Delhi, 2007.

[10] G. D. Smith. "Numerical Solution of Partial Differential Equations". Third Edition. Oxford University Press, New York, 1985.

[11] C. A. G. Fletcher. "Computational Techniques for Fluid Dynamics 1". Second Edition. Springer-Verlag, Berlin, 1990.

[12] J. Noye. Finite difference techniques for partial differential equations. In J. Noye, editor, Computational Techniques for Differential Equations, Vol. 83, pages 95-354. Elsevier, 1984.

Publish your research article in AIJR journals-

$\checkmark$ Online Submission and Tracking

$\checkmark$ Peer-Reviewed

$\checkmark$ Rapid decision

$\checkmark$ Immediate Publication after acceptance

$\checkmark$ Articles freely available online

$\checkmark$ Retain full copyright of your article.

Submit your article at journals.aijr.in 